\title{
A SIMPLE TREATMENT OF THE PROBLEM OF RADIATIVE TRANSFER IN
} SUPERNOVA-LIKE ENVELOPES

$$
\text { J. Isern }{ }^{1,2} \text {. R.Lónez }{ }^{2,3} \text { and E.Simonneau* }
$$

1) Instituto de Astrofisica de Andalucia(C.S.I.C.) Apdo.2144. 18080 Granada (Spain).2) Grup d"Astrofisica (Institut d'Estudis Catalans). 3) Debartament de Fisica de l"Atmosfera. Astronomia i Astroflsica. (Universitat de Barcelona) Diagonal 647. OBO2B Barcalona (Spain). 4) Institut d"Astrophysique de Paris 9 bbis Bd. Aragó. 75014 Paris (France).

Since the only direct information. on the physics of supernova explosions comes fram spectrophotometry of their light curves, it is wortwhile to put considerable effort on understanding the properties of supernova atmospheres. Two problems appear, however, in doing so. First, the mean free path of a photon in the region where the soectrum forms (the atmosphere) is an important fraction of the supernova size and the plane parallel approximation breaks down. Second, the roles of scattering and absorption in the formation of the continuum are not clearly determined.

The complications introduced by spherical geometry arise from the angular derivative terms in the transfer eauation and from the hioh degree of anisotropy intraduced by the geometry. The comolications introduced by scattering arise from the presence of the mean intensity in the source term of the transfer equation. As an outcome,its numerical solution is time consuming, and classical simplifications are not possible due to the strong anisotropy of the radiation field. In hydrodynamical calculations it is better to calculate the moments of the radiation field, but the success of this method depends on the possibility of replacing the infinite set of differential equations by the equations of low order moments plus a closure relationship that keeps all the properties of the higher moments.

The equations for the moments (frequency inteorated) are, in the comoving frame (Castor 1972):

$$
\begin{aligned}
& \frac{1}{C} \frac{\partial J}{\partial t}+4 \pi r^{2} \rho \frac{\partial H}{\partial M_{r}}+\frac{2 H}{r}-(3 K-J) \frac{V}{C r}-(J+K) \frac{1}{C} \frac{\partial I n \rho}{\partial t}=-\int_{0}^{\infty}\left(k_{\nu} J \nu-j_{\nu}\right) d \nu \\
& \frac{1}{C} \frac{\partial H}{\partial t}+4 \pi r^{2} \rho \frac{\partial K}{\partial M_{r}}+\frac{3 K-J}{r}-\frac{2 V}{C r} H-\frac{2}{C} \frac{\partial 1 n \rho}{\partial t} H=-\int_{0}^{\infty} k_{\nu} H_{\nu} d \nu
\end{aligned}
$$


which take into account the geometrical dilution of the eneroy and momentum fluxes. In order to solve both equations it is necessary to have a linear relationship between the moments appearing in both equations.

As the intensity strongly peaks inside the cone $\mu \leq \mu_{c}$ subtended by the opaque interior, the Eddington relationship is no longer valid since it lies on the hypothesis that the intensity is quasiisotrapic in the regions $(0<\mu<1)$ and $(-1<\mu<0)$. However, this relationstip can be easily generalized to the spherical case by defining a two zone model ( $\mu \leq \mu_{c} ; \mu>\mu_{c}$ ) (Simonneau 1980; Isern, López, Simonneau 1987; López, Simonneau, Isern 1987; Simonneau, Isern, López 1987). This two region model gives the relationship:

$3 \mathrm{~K}-\mathrm{J}=2 \mu \mathrm{H}$

$\frac{d \mu_{c}}{d r}=\frac{1-\mu_{c}^{2}}{r \mu_{c}} e^{-\tau / \mu_{c}}$

with the initial condition $\mu_{c}=0$ for $r=0$. It is easy to see that (1) and (2) with the boundary conditions: $r=0, H=0 ; \quad r=R, H=\frac{1}{2} J\left(1+\mu_{c}\right)$ and the linear relationship (3) give the correct behaviour at the center and at the outer boundary of the envelope (López, Simonneau, Isern 1987).

Equation (4) only describes the anisotropy due to the geometry and the opacity distribution, but not that introduced by the scale height of the source function. This means that the accuracy of the solutions obtained by the method proposed here improves when temperature gradients are smouth or/and scattering is dominant. The accuracy, in the worst case, for a supernovalike envelope is better than $10 \%$ in J for $\tau<1$, and better than $5 \%$ in $H(\tau=0)$.

This work has been supported by the "Subdirección General de Cooperación Científica y Técnica", the CAICYT grant 400-84 and the CSIC progran Nuclear Astrophysics.

\section{REFERENCES}

Castor, J.I., 1972, Astrophys. J.,178, 779.

Isern,J., López,R., and Simonneau,E., 1987, Rev.Mex.Astron. Astrof., 14 in press.

López,R., Simonneau,E., and Isern,J., 1987, Astr. Astroph., 184, in press.

Simonneau, E. , Isern, J., and López, R., 1987, preprint.

Simonneau,E., 1980, Bul1 . Sac. Roy. des Sci. de Liège.n9-10, p.281. 